\title{
MEASUREMENT OF NATURAL TECTONIC STATE OF STRESS IN ROCK FOUNDATION BY BACK CALCULATION METHOD WITH DEFORMATION OF TUNNEL CONTOUR
}

\author{
NGHIEM HUU HANH \\ Institute for Water Resources Research, Vietnam
}

\section{Introduction}

When designing construction in rock foundation, measurement of natural state of stress in rock mass is an important problem. The state of stress of rock foundation is defined by gravitational and tectonic force fields. The gravitational force field can not be separated from the rock materials. At any point in an elastic homogeneous isotropic rock mass gravitational components of stress field are defined by the formulas:

$$
\begin{aligned}
& \sigma_{z}^{0}=\sigma_{1}^{0}=\gamma H, \\
& \sigma_{x}^{0}=\sigma_{y}^{0}=\sigma_{2}^{0}=\sigma_{3}^{0}=\lambda \gamma H
\end{aligned}
$$

where

$\sigma_{z}^{0}, \sigma_{x}^{0}, \sigma_{y}^{0}$ - vertical and horizontal stresses of the point $\mathrm{A}$;

$H$ - depth of the point $\mathrm{A}$;

$\gamma$ - volumetric weight of the overlaying rock layers of the point $A_{;}$

$\lambda$ - coefficient of lateral pressure of rock mass at rest.

The tectonic force field is significantly more complex than gravitational force field. It is related to the complex and non-uniform distribution of the rates of tectonic movement and the rate of strain of the earth crust. There are some methods for measurement of tectonic state of stress in rock mass. In this paper author represent a method of back calculation with deformations of tunnel contour that are measured in tunneling process.

\section{Main idea of the method}

The tectonic component of stress field results from the continuous redistribution in space of the tectonic movement and deformation rate of earth crust. It takes place in rock mass of tectonic zone and in igneous and metamorphic rock mass. When an underground opening is introduced in foundation, natural tectonic state of stress is redistributed. The total stress around an opening is resulted from induced stress called by man made activities and virgin stress or in-situ stress. It is changed in dependency on distance from the tunnel face to a point in question. In figure 1 we have two perpendicular circular tunnels $\mathrm{P}$ and $\mathrm{Q}$. Tunnel $\mathrm{P}$ is directed to axis $O X$ and tunnel $\mathrm{Q}$ to axis $O Y$. At section A-A (tunnel P) and at section C-C (tunnel Q) near the face of tunnel six points are fixed according to three directions 1, 2 and 3 (figure 1). Just, the relationship between stress and strain is termed a state of a plane stresses. When the tunnel face gets a position B-B 
(tunnel P) and D-D (tunnel Q), the distances AB and CD are bigger than the diameter of tunnel. We have condition with plane deformation. If the values of deformation increment of fixed points are measured, natural tectonic state of stress can be calculated by the elastic theory.

3. Basic equation and solution

\subsection{Analysis of stress - strain relationship}

In figure 1, let A-A of tunnel $P$ and $\mathrm{C}-\mathrm{C}$ of tunnel $\mathrm{Q}$ be the sections interested.
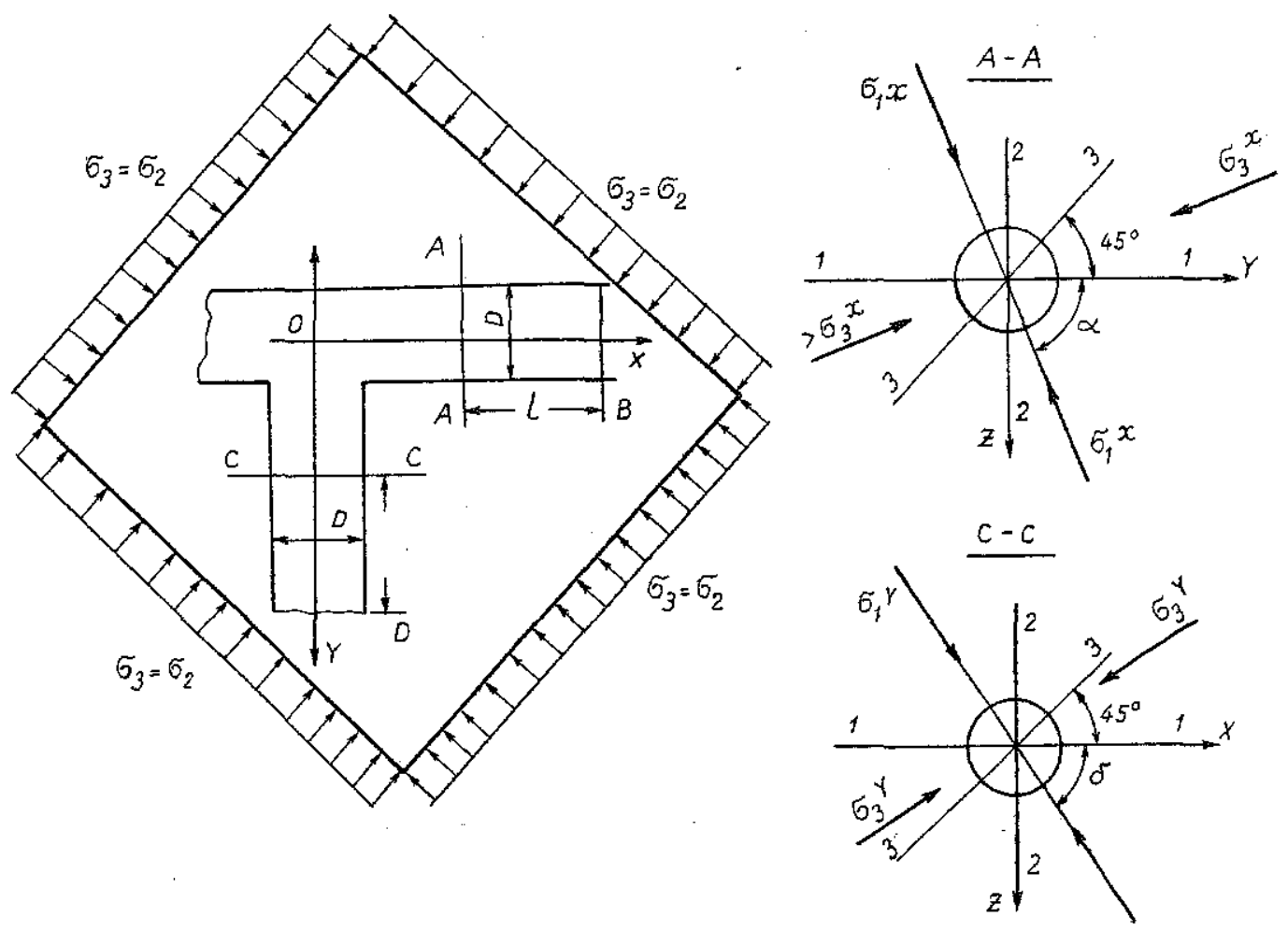

Fig. 1. Illustration of tectonic state of stresses in rock mass rounding tunnel

When A-A and C-C are next to face of tunnel major and minor stresses, after [1], they can be calculated as follows:

At section A-A:

$$
\begin{aligned}
& \sigma_{1}^{A 1}=k_{1} \sigma_{1}^{x}+k_{2} \sigma_{3}^{x}+k_{0} \sigma_{y}^{x}, \\
& \sigma_{3}^{A 1}=k_{2} \sigma_{1}^{x}+k_{1} \sigma_{3}^{x}+k_{0} \sigma_{y}^{x},
\end{aligned}
$$

At section C-C:

$$
\begin{aligned}
& \sigma_{1}^{C 1}=k_{1} \sigma_{1}^{y}+k_{2} \sigma_{3}^{y}+k_{0} \sigma_{x}^{y}, \\
& \sigma_{3}^{C 1}=k_{2} \sigma_{1}^{y}+k_{1} \sigma_{3}^{y}+k_{0} \sigma_{x}^{y},
\end{aligned}
$$

where

$\sigma_{1}^{A 1}, \sigma_{3}^{A 1}$ - average major and minor normal stresses at section A-A, $\sigma_{1}^{C 1}, \sigma_{3}^{C 1}$ - average major and minor normal stresses at section C-C, 
$\sigma_{1}^{x}, \sigma_{3}^{x}$ - projection of major and minor normal stress in the plane which is perpendicular to the direction of tunnel axis $O X$;

$\sigma_{1}^{y}, \sigma_{3}^{y}$ - projection of major and minor normal stress in the plane which is perpendicular to the direction of tunnel axis $O Y$;

$\sigma_{y}^{x}$ - normal stress in direction $O X$ of tunnel $\mathrm{P}$;

$\sigma_{x}^{y}$ - normal stress in direction $O Y$ of tunnel $\mathrm{Q}$;

$k_{1}$ - coefficient of pressure concentration in the horizontal direction 1 ;

$k_{2}$ - coefficient of pressure concentration in the vertical direction 3;

$k_{0}$ - coefficient of pressure concentration in the direction of tunnel axis.

Coefficients of stress concentration can be determined by the data [1] that are shown in Fig. 2.

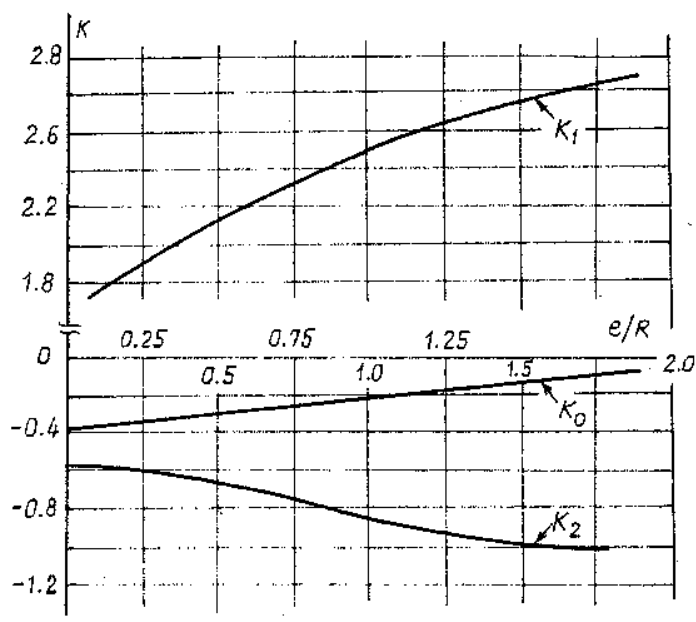

Fig. 2. Relationship between coefficient of the pressure concentration and the distance from the section to the tunnel face

At section A-A the relationships between stress and strain according to direction 1,2 and 3 are:

$$
\begin{aligned}
& \frac{U_{1}^{A 1}}{D}=\frac{1}{2 E}\left[\left(\sigma_{1}^{A 1}+\sigma_{3}^{A 1}\right)(1-\mu)+(1+\mu) \cos 2 \alpha\left(\sigma_{1}^{A 1}-\sigma_{3}^{A 1}\right)\right], \\
& \frac{U_{2}^{A 1}}{D}=\frac{1}{2 E}\left[\left(\sigma_{1}^{A 1}+\sigma_{3}^{A 1}\right)(1-\mu)+(1+\mu) \cos 2\left(\alpha+45^{\circ}\right)\left(\sigma_{1}^{A 1}-\sigma_{3}^{A 1}\right)\right], \\
& \frac{U_{3}^{A 1}}{D}=\frac{1}{2 E}\left[\left(\sigma_{1}^{A 1}+\sigma_{3}^{A 1}\right)(1-\mu)+(1+\mu) \cos 2\left(\alpha+90^{\circ}\right)\left(\sigma_{1}^{A 1}-\sigma_{3}^{A 1}\right)\right],
\end{aligned}
$$

where:

$U_{1}^{A 1}, U_{2}^{A 1}, U_{3}^{A 1}$ - the radial displacements of tunnel contour according to direction 1,2 and 3 at section A-A near the face tunnel;

$D$ - diameter of tunnel cross section;

$E$ - Young's modulus;

$\mu$ - Poisson's ratio. 
The substitution of the terms $\sigma_{1}^{A 1}$ and $\sigma_{3}^{A 1}$ from equation (3.1) into equation (3.3) gives

$$
\begin{aligned}
& U_{1}^{A 1}=\frac{D}{2 E}\left[\left(\sigma_{1}^{x}+\sigma_{3}^{x}\right)\left(k_{1}+k_{2}\right)(1-\mu)+(1+\mu)\left(k_{1}-k_{2}\right) \cos 2 \alpha\left(\sigma_{1}^{x}-\sigma_{3}^{x}\right)+C \sigma_{y}^{x}\right], \\
& U_{2}^{A 1}=\frac{D}{2 E}\left[\left(\sigma_{1}^{x}+\sigma_{3}^{x}\right)\left(k_{1}+k_{2}\right)(1-\mu)+(1+\mu)\left(k_{1}-k_{2}\right) \cos 2\left(\alpha+45^{\circ}\right)\left(\sigma_{1}^{x}-\sigma_{3}^{x}\right)+C \sigma_{y}^{x}\right], \\
& U_{3}^{A 1}=\frac{D}{2 E}\left[\left(\sigma_{1}^{x}+\sigma_{3}^{x}\right)\left(k_{1}+k_{2}\right)(1-\mu)+(1+\mu)\left(k_{1}+k_{2}\right) \cos 2\left(\alpha+90^{\circ}\right)\left(\sigma_{1}^{x}-\sigma_{3}^{x}\right)+C \sigma_{y}^{x}\right],
\end{aligned}
$$

where $C=(D / E) k_{0}(1-\mu)$.

Making the same calculation with section $\mathrm{C}-\mathrm{C}$ according to direction 1,2 and 3 we have the radial displacements at section $\mathrm{C}-\mathrm{C}$ as follows:

$$
\begin{aligned}
U_{1}^{C 1}= & \frac{D}{2 E}\left[\left(\sigma_{1}^{y}+\sigma_{3}^{y}\right)\left(k_{1}+k_{2}\right)(1-\mu)\right. \\
& \left.+(1+\mu)\left(k_{1}-k_{2}\right) \cos 2 \delta\left(\sigma_{1}^{y}-\sigma_{3}^{y}\right)+C \sigma_{x}^{y}\right], \\
U_{2}^{C 1}= & \frac{D}{2 E}\left[\left(\sigma_{1}^{y}+\sigma_{3}^{y}\right)\left(k_{1}+k_{2}\right)(1-\mu)\right. \\
& \left.+(1+\mu)\left(k_{1}-k_{2}\right) \cos 2\left(\delta+45^{\circ}\right)\left(\sigma_{1}^{y}-\sigma_{3}^{y}\right)+C \sigma_{x}^{y}\right], \\
U_{3}^{C 1}= & \frac{D}{2 E}\left[\left(\sigma_{1}^{y}+\sigma_{3}^{y}\right)\left(k_{1}+k_{2}\right)(1-\mu)\right. \\
& \left.+(1+\mu)\left(k_{1}+k_{2}\right) \cos 2\left(\delta+90^{\circ}\right)\left(\sigma_{1}^{y}-\sigma_{3}^{y}\right)+C \sigma_{x}^{y}\right],
\end{aligned}
$$

where:

$U_{1}^{C 1}, U_{2}^{C 1}, U_{3}^{C 1}$ - the radial displacements of tunnel contour according to direction 1,2 and 3 at position $\mathrm{C}-\mathrm{C}$ near the face of tunnel;

When the faces of tunnel have been advanced sections A-A and C-C about one time the tunnel diameter beyond section A-A and C-C, stress-strain relationship at section A-A and C-C are termed a state of plane deformation. The deformations of tunnel contour according to direction 1,2 and 3 are calculated by:

at section A-A:

$$
\begin{aligned}
& \frac{U_{1}^{A 2}}{D}=\frac{1-\mu^{2}}{2 E}\left[\left(\sigma_{1}^{x}+\sigma_{3}^{x}\right)+\cos 2 \alpha\left(\sigma_{1}^{x}-\sigma_{3}^{x}\right)\right], \\
& \frac{U_{2}^{A 2}}{D}=\frac{1-\mu^{2}}{2 E}\left[\left(\sigma_{1}^{x}+\sigma_{3}^{x}\right)+\cos 2\left(\alpha+45^{\circ}\right)\left(\sigma_{1}^{x}-\sigma_{3}^{x}\right)\right], \\
& \frac{U_{3}^{A 2}}{D}=\frac{1-\mu^{2}}{2 E}\left[\left(\sigma_{1}^{x}+\sigma_{3}^{x}\right)+\cos 2\left(\alpha+90^{\circ}\right)\left(\sigma_{1}^{x}-\sigma_{3}^{x}\right)\right],
\end{aligned}
$$

at section $\mathrm{C}-\mathrm{C}$

$$
\begin{aligned}
& \frac{U_{1}^{C 2}}{D}=\frac{1-\mu^{2}}{2 E}\left[\left(\sigma_{1}^{y}+\sigma_{3}^{y}\right)+\cos 2 \delta\left(\sigma_{1}^{y}-\sigma_{3}^{y}\right)\right], \\
& \frac{U_{2}^{C 2}}{D}=\frac{1-\mu^{2}}{2 E}\left[\left(\sigma_{1}^{y}+\sigma_{3}^{y}\right)+\cos 2\left(\delta+45^{\circ}\right)\left(\sigma_{1}^{y}-\sigma_{3}^{y}\right)\right] \\
& \frac{U_{3}^{C 2}}{D}=\frac{1-\mu^{2}}{2 E}\left[\left(\sigma_{1}^{y}+\sigma_{3}^{y}\right)+\cos 2\left(\delta+90^{\circ}\right)\left(\sigma_{1}^{y}-\sigma_{3}^{y}\right)\right]
\end{aligned}
$$

where: 
$U_{1}^{A 2}, U_{2}^{A 2}, U_{3}^{A 2}$ - the radial displacements of the tunnel contour according to direction 1,2 and 3 at section $\mathrm{A}-\mathrm{A}$ when $A B>D$;

$U_{1}^{C 2}, U_{2}^{C 2}, U_{3}^{C 2}$ - the radial displacements of the tunnel contour according to direction 1,2 and 3 at section $\mathrm{C}-\mathrm{C}$ when $C D>D$.

\subsection{Analysis of in-situ stresses}

Substituting equation (3.6) into (3.4) gives

$$
\begin{aligned}
U_{1}^{x}= & \frac{D}{2 E}\left\{\sigma_{1}^{x}\left[2\left(1-\mu^{2}\right)-\left(k_{1}+k_{2}\right)(1-\mu)\right]+\sigma_{3}^{x}\left[2\left(1-\mu^{2}\right)-\left(k_{1}+k_{2}\right)(1-\mu)\right]\right. \\
& \left.-\sigma_{1}^{x} \cos 2 \alpha\left[4\left(1-\mu^{2}\right)-\left(k_{1}-k_{2}\right)(1+\mu)\right]+\sigma_{3}^{x} \cos 2 \alpha\left[4\left(1-\mu^{2}\right)-\left(k_{1}-k_{2}\right)(1+\mu)\right]\right\}, \\
U_{2}^{x}= & \frac{D}{2 E}\left\{\sigma_{1}^{x}\left[2\left(1-\mu^{2}\right)-\left(k_{1}+k_{2}\right)(1-\mu)\right]+\sigma_{3}^{x}\left[2\left(1-\mu^{2}\right)-\left(k_{1}+k_{2}\right)(1-\mu)\right]\right. \\
& \left.-\sigma_{1}^{x} \sin 2 \alpha\left[4\left(1-\mu^{2}\right)-\left(k_{1}-k_{2}\right)(1+\mu)\right]+\sigma_{3}^{x} \sin 2 \alpha\left[4\left(1-\mu^{2}\right)-\left(k_{1}-k_{2}\right)(1+\mu)\right]\right\}, \\
U_{3}^{x}= & \frac{D}{2 E}\left\{\sigma_{1}^{x}\left[2\left(1-\mu^{2}\right)-\left(k_{1}+k_{2}\right)(1-\mu)\right]+\sigma_{3}^{x}\left[2\left(1-\mu^{2}\right)-\left(k_{1}+k_{2}\right)(1-\mu)\right]\right. \\
& \left.+\sigma_{1}^{x} \cos 2 \alpha\left[4\left(1-\mu^{2}\right)-\left(k_{1}-k_{2}\right)(1+\mu)\right]+\sigma_{3}^{x} \cos 2 \alpha\left[4\left(1-\mu^{2}\right)-\left(k_{1}-k_{2}\right)(1+\mu)\right]\right\},
\end{aligned}
$$

where:

$$
\begin{gathered}
U_{1}^{x}=U_{1}^{A 2}-U_{1}^{A 1}-C \sigma_{y}^{x}, \\
U_{2}^{x}=U_{2}^{A 2}-U_{2}^{A 1}-C \sigma_{y}^{x}, \\
U_{3}^{x}=U_{3}^{A 2}-U_{3}^{A 1}-C \sigma_{y}^{x}, \\
A+B=2\left(1-\mu^{2}\right)-\left(k_{1}+k_{2}\right)(1-\mu), \\
A-B=4\left(1-\mu^{2}\right)-\left(k_{1}-k_{2}\right)(1+\mu) .
\end{gathered}
$$

Calculating equations (3.10) gives

$$
\begin{aligned}
& A=3\left(1-\mu^{2}\right)-k_{1}+\mu k_{2}, \\
& B=\mu^{2}-1-k_{2}+\mu k_{1} .
\end{aligned}
$$

Substitution of $A$ and $B$ from equation (3.11) into equation (3.8) gives

$$
\begin{aligned}
& U_{1}^{x}=\frac{D}{2 E}\left[\sigma_{1}^{x}(A+B)+\sigma_{3}^{x}(A+B)-\sigma_{1}^{x} \cos 2 \alpha(A-B)+\sigma_{3}^{x} \cos 2 \alpha(A-B)\right], \\
& U_{2}^{x}=\frac{D}{2 E}\left[\sigma_{1}^{x}(A+B)+\sigma_{3}^{x}(A+B)-\sigma_{1}^{x} \sin 2 \alpha(A-B)+\sigma_{3}^{x} \sin 2 \alpha(A-B)\right] \\
& U_{3}^{x}=\frac{D}{2 E}\left[\sigma_{1}^{x}(A+B)+\sigma_{3}^{x}(A+B)+\sigma_{1}^{x} \cos 2 \alpha(A-B)-\sigma_{3}^{x} \cos 2 \alpha(A-B)\right] .
\end{aligned}
$$

The solution of the equation (3.12) will be:

$$
\begin{aligned}
\sigma_{1,3}^{x} & =\frac{E}{D}\left[\frac{U_{1}^{x}+U_{3}^{x}}{2(A+B)} \pm \frac{U_{3}^{x}-U_{1}^{x}}{2(A-B)} \sqrt{1+\operatorname{tg}^{2} 2 \alpha}\right] \\
\operatorname{tg} 2 \alpha & =\frac{2 U_{2}^{x}-\left(U_{1}^{x}+U_{3}^{x}\right)}{U_{1}^{x}-U_{3}^{x}}
\end{aligned}
$$


A similar calculation for section C-C gives the component of stresses $\sigma_{1,3}^{y}$ and the angle $\delta$ :

$$
\begin{aligned}
\sigma_{1,3}^{y} & =\frac{E}{D}\left[\frac{U_{1}^{y}+U_{3}^{y}}{2(A+B)} \pm \frac{U_{3}^{y}-U_{1}^{y}}{2(A-B)} \sqrt{1+\operatorname{tg}^{2} 2 \delta}\right] \\
\operatorname{tg} 2 \delta & =\frac{2 U_{2}^{y}-\left(U_{1}^{y}+U_{3}^{y}\right)}{U_{1}^{x}-U_{3}^{x}}
\end{aligned}
$$

where:

$$
\begin{aligned}
& U_{1}^{y}=U_{1}^{C 2}-U_{1}^{C 1}-C \sigma_{x}^{y}, \\
& U_{2}^{y}=U_{2}^{C 2}-U_{2}^{C 1}-C \sigma_{x}^{y}, \\
& U_{3}^{y}=U_{3}^{A 2}-U_{3}^{A 1}-C \sigma_{x}^{y} .
\end{aligned}
$$

Equations (3.13) and (3.14) will be determined if $\sigma_{y}^{x}$ and $\sigma_{x}^{y}$ are measured. In a different way, for calculating $\sigma_{1,3}^{x}$ and $\sigma_{1,3}^{y}$ we have to determine increment of the displacement of tunnel contour that are shown in equation (3.9) and equation (3.15).

From equations (3.13) and (3.14) we have tectonic components of stress field in two dimensions $O X$ and $O Y$ as follows:

$$
\begin{aligned}
\sigma_{x}^{y} & =\frac{E}{D}\left(\frac{U_{1}^{y}+U_{3}^{x}}{A+B}-\frac{U_{3}^{y}-U_{1}^{x}}{A-B}\right), \\
\sigma_{y}^{x} & =\frac{E}{D}\left(\frac{U_{1}^{x}+U_{3}^{x}}{A+B}-\frac{U_{3}^{x}-U_{1}^{x}}{A-B}\right) .
\end{aligned}
$$

Substituting values of displacement of tunnel contour from equations (3.9) and (3.15) into the equation (3.16) gives

$$
\begin{aligned}
& \sigma_{y}^{x}=\frac{2 E}{D} \frac{U_{1}^{C 2}-U_{3}^{C 1}-2 k_{0}(1-\mu)\left(U_{1}^{A 2}-U_{1}^{A 1}\right)}{\left[2 k_{0}(1-\mu)\right]^{2}+1}, \\
& \sigma_{x}^{y}=\frac{2 E}{D} \frac{U_{1}^{A 2}-U_{3}^{A 1}-2 k_{0}(1-\mu)\left(U_{1}^{C 2}-U_{1}^{C 1}\right)}{\left[2 k_{0}(1-\mu)^{2}\right]+1} .
\end{aligned}
$$

With the terms $\sigma_{y}^{x}$ and $\sigma_{x}^{y}$ from (3.17), equations (3.9) and (3.15) give:

$$
\begin{aligned}
& U_{1}^{x}=U_{1}^{A 2}-U_{1}^{A 1}-U_{0}^{A x}, \\
& U_{2}^{x}=U_{2}^{A 2}-U_{2}^{A 1}-U_{0}^{A x}, \\
& U_{3}^{x}=U_{3}^{A 2}-U_{3}^{A 1}-U_{0}^{A x},
\end{aligned}
$$

and

$$
\begin{aligned}
& U_{1}^{y}=U_{1}^{C 2}-U_{1}^{C 1}-U_{0}^{C y} \\
& U_{2}^{y}=U_{2}^{C 2}-U_{2}^{C 1}-U_{0}^{C y} \\
& U_{3}^{y}=U_{3}^{A 2}-U_{3}^{A 1}-U_{0}^{C y}
\end{aligned}
$$

where

$$
\begin{aligned}
& U_{0}^{A x}=2 k_{0}(1-\mu) \frac{U_{1}^{C 2}-U_{1}^{C 1}-2 k_{0}(1-\mu)\left(U_{1}^{A 2}-U_{1}^{A 1}\right)}{\left[2 k_{0}(1-\mu)\right]^{2}+1}, \\
& U_{0}^{C y}=2 k_{0}(1-\mu) \frac{U_{1}^{A 2}-U_{1}^{A 1}-2 k_{0}(1-\mu)\left(U_{1}^{C 2}-U_{1}^{C 1}\right)}{\left[2 k_{0}(1-\mu)\right]^{2}+1} .
\end{aligned}
$$


Orientation and value of major and minor normal stresses are shown in Fig. 3.

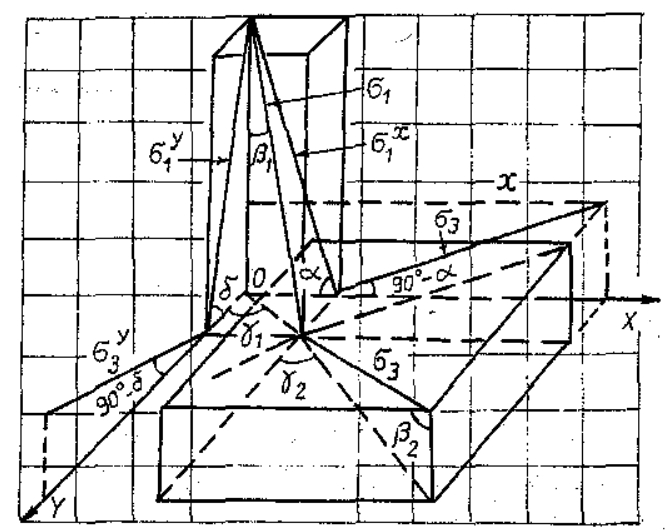

Fig. 3. Illustration of value and orientation of major and minor normal stresses in the tectonic force field

From Fig. 3 we have

$$
\begin{gathered}
\sigma_{1}=\sqrt{\left(\sigma_{1}^{y}\right)^{2}+\left(\sigma_{1}^{x}\right)^{2} \times \frac{1+\operatorname{tg}^{2} \delta}{1+\operatorname{tg}^{2} \alpha}}, \\
\operatorname{tg} \beta_{1}=\sqrt{\operatorname{tg} \alpha-\frac{\left(\sigma_{1}^{y}\right)^{2} \operatorname{tg}^{2} \delta\left(1+\operatorname{tg}^{2} \alpha\right)}{\left(\sigma_{1}^{x}\right)^{2}\left(1+\operatorname{tg}^{2} \delta\right)}} \\
\operatorname{tg} \gamma_{1}=\frac{\sigma_{1}^{x} \operatorname{tg} \alpha}{\sigma_{1}^{y} \operatorname{tg} \delta} \sqrt{\frac{1+\operatorname{tg}^{2} \delta}{1+\operatorname{tg}^{2} \alpha}} \\
\sigma_{3}=\sqrt{\frac{\left(\sigma_{3}^{y}\right)^{2}+\left(\sigma_{3}^{x}\right)^{2} \times \frac{1+\operatorname{cotg}^{2} \alpha}{1+\operatorname{cotg}^{2} \delta}}{\operatorname{tg} \beta_{2}=}} \\
\operatorname{tg}{ }_{\operatorname{cotg} \alpha-\frac{\left(\sigma_{3}^{y}\right)^{2} \operatorname{cotg}^{2} \delta\left(1+\operatorname{cotg}^{2} \alpha\right)}{\left(\sigma_{3}^{x}\right)^{2}\left(1+\operatorname{cotg}^{2} \delta\right)}}^{=\frac{\sigma_{3}^{x} \operatorname{cotg} \alpha}{\sigma_{3}^{y} \operatorname{cotg} \delta} \cdot \sqrt{\frac{1+\operatorname{cotg}^{2} \delta}{1+\operatorname{cotg}^{2} \alpha}}}
\end{gathered}
$$

\section{Conclusion}

There exists the tectonic state of stress in the tectonic zone and in the rock foundation of igneous and metamorphic origin. It will be distributed and redistributed due to the excavation of the underground construction. Therefore, measurement of in-situ stress is very essential. The method presented can be used in the tunneling process and should be considered for correction of the existing design. 


\section{References}

1. Blokh H. P., Zubkov A. B., Feklistov Iu. G. Partial unloading method on large size. Proceedings of IGD CO AN USSR. Diagnostics of stress state of rock masses. Novosibirk. 1980 (in Russian).

2. Franklin J. A., Dusseault M. B. Rock Engineering. Mc. Graw-Hill Publishing Company. Singapore, 1989.

3. Kuberan R. Measurement of in-situ stresses in rock mass. workshop on Rock Mechanics, New Delhi. Feb. 1992.

4. Nghiem Huu Hanh. Some consideration of the constructions on and in rock mass. Proceedings of the Japan-Vietnam seminar on Geotechnical Engineering. Hanoi, March 1996 (in Vietnamese).

Received September 5, 1996

\section{XÁC ĐỊNH TRANG THÁI ỨNG SUẤT TỰ NHIÊN CỬ NỀN ĐÁ BẰNG PHƯớNG PHÁP TÍNH NGỰ̛C TƯ BIẾN DẠNG CỦA CHU TUYẾN TUNNEL}

Trạng thái ứng suất trong khối đá quanh tunnel thay đổi phụ thuộc vào khoảng cách giữa gương tunnel và mặt cắt nghiên cứu. Khi mặt cắt ớ sát gương tunnel, trạng thái ứng suất - biến dạng được phân tích theo bài toán ứng suất phẳng. Khi mặt căt cách xa gương tunnel, trạng thái ứng suất - biến dạng được xem xét trong bài toán biến dạng phẳng. Vận dụng đặc điểm đó, trong bài này tác giá kiến nghị cách xác định trạng thái ứng suất tự nhiên trong nền đá băng phương pháp tính ngược từ chuyển vị cửa chu tuyến hai tunnel vuông góc nhau. 